\title{
Ultrafast Conductivity Dynamics in Pentacene Probed using Terahertz Spectroscopy
}

\author{
V. K. Thorsmølle A R. D. Averitt, X. Chi, D. J. Hilton, D. L. Smith, A. P. Ramirez, and A. J. Taylor \\ Los Alamos National Laboratory, Los Alamos, NM 87545.
}

\begin{abstract}
We present measurements of the transient photoconductivity in pentacene single crystals using optical-pump THz-probe spectroscopy. We have measured the temperature and fluence dependence of the mobility of the photoexcited charge carriers with picosecond resolution. The pentacene crystals were excited at $3.0 \mathrm{eV}$ which is above the bandgap of $\sim 2.2 \mathrm{eV}$ and the induced change in the far-infrared transmission was measured. At $30 \mathrm{~K}$, the carrier mobility is $\mu \approx 0.4 \mathrm{~cm}^{2} / \mathrm{Vs}$ and decreases to $\mu \approx 0.2 \mathrm{~cm}^{2} / \mathrm{Vs}$ at room temperature. The transient terahertz signal reveals the presence of free carriers that are trapped on the timescale of a few ps or less, possibly through the formation of excitons, small polarons, or trapping by impurities.
\end{abstract}

The promise of organic semiconductors for technological applications in electronics and photonics has spurred an immense interest in these materials $\stackrel{1}{1}$. However, despite intense research efforts in recent years, the nature of charge transport and photoexcitations in conjugated polymers and organic molecular crystals is still not well understood and remains controversial 2 .

Conductivities in undoped organic semiconducters are orders of magnitude smaller than in inorganic semiconducters such as $\mathrm{Si}$ and GaAs. Polyacenes such as naphthalene $(\mathrm{Nph})$ and pentacene $(\mathrm{Pc})$ are conjugated molecules with mobilities, $\mu$, on the order of $1 \mathrm{~cm}^{2} / \mathrm{Vs}$. The main contributing factors to such small mobilities are thought to be related to weak intermolecular interactions. These factors include strong localization, polaron formation and trapping by impurities. However, there is little firm experimental evidence for the importance of any single one of these factors in single crystals. The temperature $(T)$ dependence of the mobility in these crystals is observed to increase as the temperature is lowered ${ }^{3}$. This has been attributed to a transition from a polaron hopping transport to a band-like transport of carriers at lower temperatures described by some theoretical models 4.5 and seen by Schlein et al. in $\mathrm{Nph}^{6}$. In some materials the temperature dependence follows $\mu \propto T^{-n}, n>0$. Warta et al. observed this behavior in Nph until low temperature, $\sim 30 \mathrm{~K}$, where it then levels off at values greater than $100 \mathrm{~cm}^{2} / \mathrm{Vs}^{3.7}$. Clearly, the nature of charge carrier transport is still not completely understood.

It is not clear either whether electronic excitations are best described by the molecular exciton model, or by the semiconductor band mode ${ }^{8}$. In the former case, the excited states are localized and the primary photoexcitations are excitons, which can dissociate into free polarons. In the latter case, they are delocalized and mobile polarons are created directly from free electronhole pairs by the absorption of light. Ultrafast optical measurements are important in this regard in that the dynamics of the photogenerated carriers can be temporally resolved. While the optical-pump probe technique is a noncontact technique and yields important information of the relaxation dynamics, optical-pump terahertzprobe is a direct probe of the nonequilibrium carrier con- ductivity dynamics. Terahertz time-domain spectroscopy (THz-TDS) without an optical pump is an ultrafast optical technique in which near single-cycle freespace electric field transients of a duration of about $1 \mathrm{ps}$ and spectral width on the order of $1 \mathrm{THz}$ are used to measure the complex conductivity of the sample. This is a coherent technique, and by varying the probe delay time with respect to an optical excitation pulse the induced conductivity changes of the sample can be measured with sub-picosecond (sub-ps) resolution. Thus, THz-TDS has become an important technique in the study of the farinfrared conductivity in condensed matter systems ranging from carrier transport in semiconductors to the dynamics of fluids $9.10,11,12,13,14,15,16,17$.

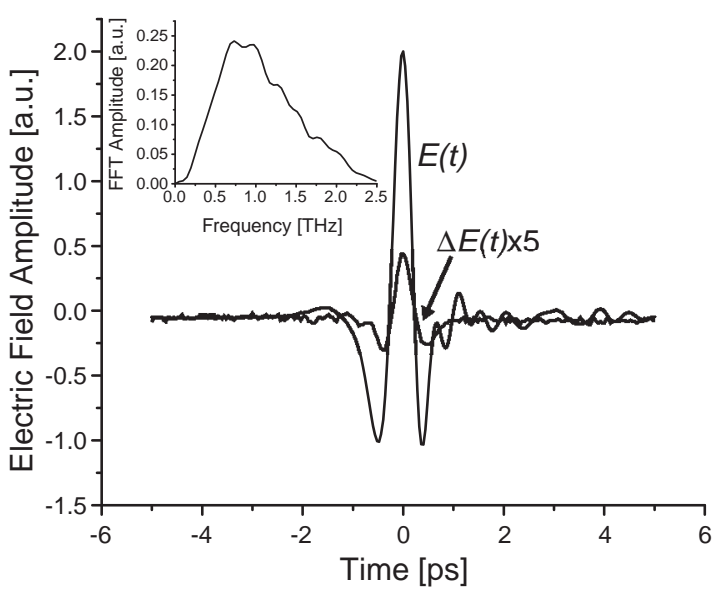

FIG. 1: Electric field $E(t)$ of $\mathrm{THz}$ pulse transmitted through Pc crystal, and induced change in electric field $\Delta E(t)$ at $T=4$ $K$. The inset shows the fast Fourier transformed amplitude spectrum of $E(t)$.

Here we present optical-pump THz-probe conductivity measurements on Pc single crystals. High quality Pc single crystals were grown in a flow of inert gas. The crystals used were typically $3 \mathrm{~mm} \times 3 \mathrm{~mm}$ and approximately 50 $\mu \mathrm{m}$ thick. The experiments utilized a commercial-based regeneratively amplified Ti: $\mathrm{Al}_{2} \mathrm{O}_{3}$ system operating at 1 $\mathrm{KHz}$ producing nominally $2.0 \mathrm{~mJ}$, sub-50 fs pulses at 1.5 $\mathrm{eV}$. The $\mathrm{THz}$ pulses were generated and detected using 
electrooptic techniques. The schematic of the THz-setup is shown in Ref 18 . The Pc crystals were here excited at $3.0 \mathrm{eV}$ which is above the bandgap of $2.2 \mathrm{eV}$. The experiments were performed in transmission with the electric field of the $\mathrm{THz}$ pulses in the $a b$-plane. The diameter of the $\mathrm{THz}$ beam is apertured to $2 \mathrm{~mm}$ at the sample position, and the diameter of the pump beam is $\sim 3 \mathrm{~mm}$. The samples were mounted inside an optical He cryostat. The results presented are obtained from several samples.

Figure 1shows the electric field of the THz pulse transmitted through a Pc crystal without optical excitation, compared to the induced change in the electric field with optical excitation. There is a decrease in the transmitted electric field associated with the conductivity of the mobile carriers (i.e. Drude-like response). No shift in the phase of the induced change of the $\mathrm{THz}$ pulse is observed. Thus it is valid to measure changes in the peak amplitude of the transmitted $\mathrm{THz}$ pulses to determine the photoinduced conductivity (i.e. $\Delta \sigma \propto \Delta E / E$ ).

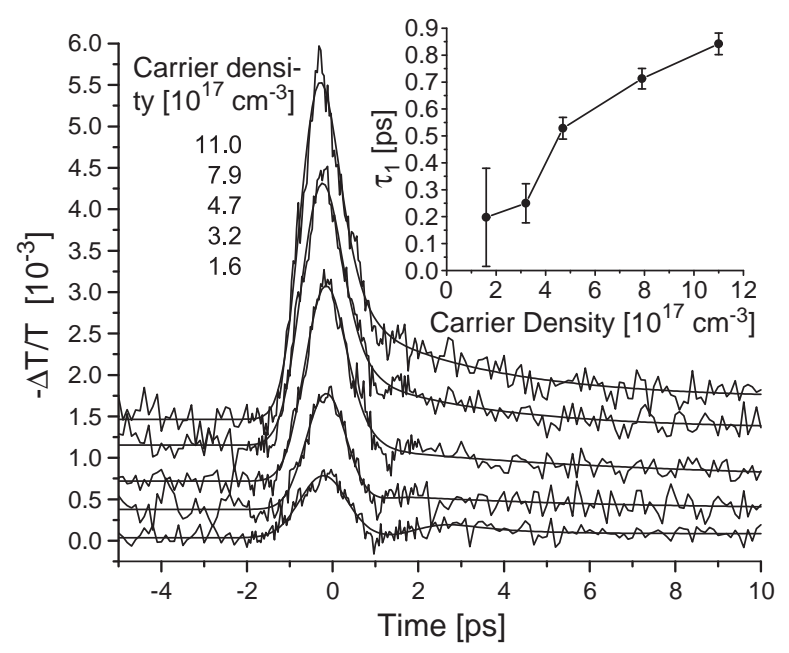

FIG. 2: Differential transmission of the peak of the THz probe pulse versus pump-probe delay time at various fluences at $T=$ $20 \mathrm{~K}$. The plots are displaced vertically for clarity. The inset shows the fast exponential decay time versus pump fluence.

Figure 2 shows the induced change in transmission of the $\mathrm{THz}$ electric field $-\Delta \mathrm{T} / \mathrm{T}$ at incident pump fluences ranging from 0.12 to $0.83 \mathrm{~mJ} / \mathrm{cm}^{2}$ producing carrier densities from $1.6 \times 10^{17}$ to $1.1 \times 10^{18} \mathrm{~cm}^{-3}$ at $T=20 \mathrm{~K}$. The photoconductivity transient increases with increasing fluence indicating an increasing conductivity with increasing carrier density. The rise time is resolution limited by the $\mathrm{THz}$ setup. The initial exponential decay increases with increasing fluence (see inset to Figure 2), indicating some interaction between the carriers creating a bottleneck effect. This behavior could also simply be due to a saturation of the trap density where carriers are trapped by defects. At higher fluences, an additional exponential relaxation component develops with a longer lifetime leveling off at $\sim 4$ ps. $-\Delta \mathrm{T} / \mathrm{T}$ is close to linear with pump fluence.
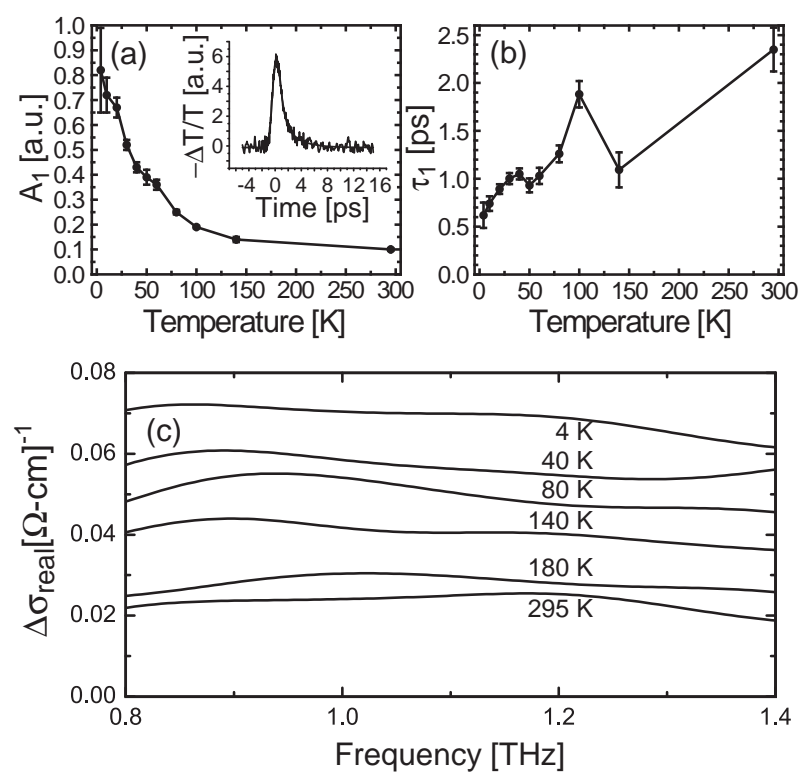

FIG. 3: Temperature dependence of various parameters at a fluence of $I=0.21 \mathrm{~mJ} / \mathrm{cm}^{2}$. (a) Fast decay amplitude versus temperature. The inset shows $-\Delta \mathrm{T} / \mathrm{T}$ at $T=4 \mathrm{~K}$. (b) Fast decay time versus temperature. (c) Induced change in real conductivity versus frequency at various temperatures.

The differential transmission versus temperature displays a similar behavior where the peak of the transient signal, and hence the transient conductivity, increases as the temperature is lowered. We attribute this to an increase in carrier mobility. Figure 3(a) shows the fast decay amplitude to be increasing with decreasing temperatures, following the mobility (see Figure 4 ). The fast decay time shown in Figure 3(b) is seen to increase with increasing temperature which is not immediately obvious, but might be due to thermal population of exciton levels or trapped states with higher temperatures. Figure 31(c) shows the induced change in the real conductivity, and it is seen to be increasing with decreasing temperatures. The induced change in the imaginary conductivity is close to zero. Thus, it was not possible to fit the data to a Drude model with such small induced conductivities.

Figure 4 shows the carrier mobility versus temperature obtained directly from the time domain data, $-\Delta \mathrm{T} / \mathrm{T}$, and the induced increase in conductivity, $\Delta \sigma=n e \mu 13$. Here, $n$ is the carrier density, $e$ is the electronic charge, and $\mu$ is the effective carrier mobility. $\Delta \sigma$ is obtained from the approximation for a thin film on a semi-infinite insulating media given by

$$
\Delta \sigma \cong-\frac{\Delta \mathrm{T}}{\mathrm{T}} \frac{(1+N)}{Z_{o} d},
$$

where $N=1.6$ is the refractive index of the media (unpumped crystal), $d=14 \mu \mathrm{m}$ is the optical penetration depth at the pump wavelength, and $Z_{o}=377 \Omega$ is 


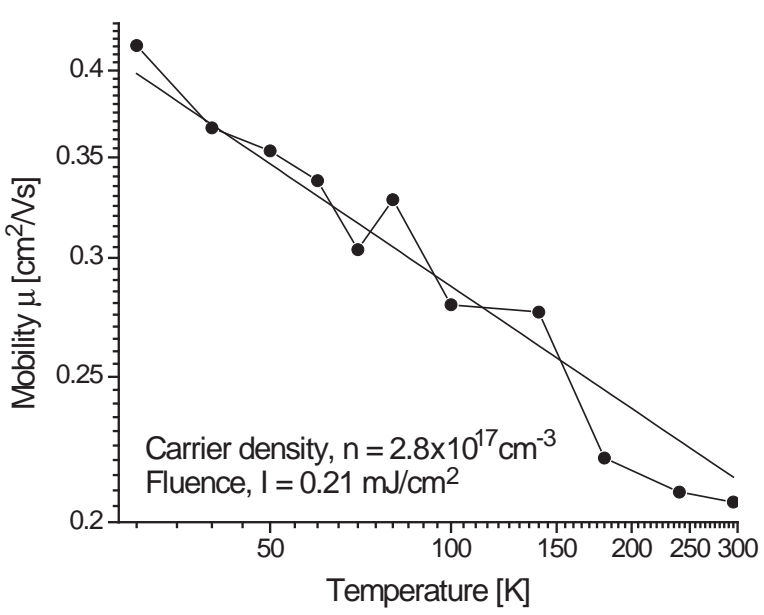

FIG. 4: Photoinduced carrier mobility versus temperature. The straight line is a fit to $T^{-n}$, where $n=0.27$.

the impedance of free space. The carrier density decays exponentially by $d$, but is assumed constant over the excitation thickness.

At low temperatures, $T=30 \mathrm{~K}$, the photoinduced carrier mobility is $\mu \approx 0.4 \mathrm{~cm}^{2} / \mathrm{Vs}$ and $\mu$ decreases to $\mu \approx 0.2 \mathrm{~cm}^{2} /$ Vs at room temperature. These values for the mobility assumes a 100\% internal quantum efficiency conversion to free carriers, which may be an overestimate of the carrier density. These results provide a lower limit on the mobility. These values are comparable to values found in Pc crystals by Bukto et al ${ }^{19}$ in a field effect geometry, as well as by Hegmann et al. also using the same technique of optical-pump THz-probe spectroscopy on functionalized Pc crystals 16 . The temperature dependence of the mobility shown in Figure 4 follows the $T^{-n}$ power-law in the temperature interval $30-300 \mathrm{~K}$, where $n=0.27 \pm 0.05$. The low value of $n$ does not give a clear indication of a pure band-like transport at low tempertures, and the continuation of the typical power-law dependence into the high-temperature regime is not completely understood ${ }^{4}$. The continuation of the power-law dependence into the high-temperature regime was also observed in Nph by Warta et al ${ }^{6.7}$. The fact that the conductivity shown in Figure 3 (c) is seen to be increasing with decreasing temperature does however indicate a band-like transport.

The fast rise time for $-\Delta \mathrm{T} / \mathrm{T}$ of $\sim 0.5$ ps suggests free carriers or polarons to be photoexcited directly, because THz-TDS is sensitive to the presence of free charge carriers. This rise time is comparable to the response time of the THz setup, and it is therefore likely that the formation of mobile charge carriers occur on a shorter timescale. This same behavior was observed by Hegmann et al. in functionalized $\mathrm{Pc}_{\mathrm{c}} \underline{16}$. Another group have reported the formation of polarons within $\sim 100 \mathrm{fs}$ in a conjugated polymer (PPV thin films) ${ }^{20.21}$, thus supporting the semiconductor band model. Yet another group finds that polarons are first generated by electric-fieldassisted dissociation of primary excitons on a timescale of $10 \mathrm{ps}$ in light emitting diodes ( $m$-LPPP thin films $)^{22}$, thus supporting the molecular exciton model. Clearly, further studies are needed to address this controversy.

In conclusion, we have measured the transient photoconductivity in $\mathrm{Pc}$ single crystals using THz-TDS. The rapid onset of photoconductivity suggests the primary photoexcitations to be mobile charge carriers. The photoinduced response consists of a two-component exponential relaxation. The initial fast component relaxes within a few ps or less, possibly through the formation of excitons, small polarons, or trapping by impurities. Photoinduced mobilities of at least $\mu=0.4 \mathrm{~cm}^{2} / \mathrm{Vs}$ at $30 \mathrm{~K}$ was obtained. The temperature dependence of the photoinduced mobilities is found to follow a power-law, $T^{-n}$, where $n=0.27$.

This research was supported by the Los Alamos Directed Research and Development Program of the U.S. Department of Energy.
* Electronic address: vthorsmolle@lanl.gov

1 M. A. Baldo, M.E. Thompson, and S.R. Forrest, Nature 403, 750 (2000).

2 F. A. Hegmann, Physics in Canada 59, 127 (2003).

${ }^{3}$ W. Warta, R. Stehle, and N. Karl, Appl. Phys. A 36, 163 (1985).

4 E. A. Silinsh, A. Klimkāns, S. Larsson and V. Čápek, Chem. Phys. 198, 311 (1995).

${ }^{5}$ E. A. Silinsh and V. Čápek, Organic Molecular Crystals: Interaction, Localization, and Transport Phenomena (American Institute of Physics, New York, 1994).

6 L. B. Schlein, C. B. Duke and A. R. McGhie, Phys. Rev. Lett. 40, 197 (1978).

7 W. Warta, and N. Karl, Phys. Rev. B 32, 1132 (1985). (American Institute of Physics, New York, 1994).

8 N. S. Sariciftci, Primary Photoexcitations in Conjugated Polymers: Molecular Exciton versus Semiconductor Band
Model World Scientific, Singapore, 1997).

9 V. K. Thorsmølle, R. D. Averitt, M. P. Maley, M. F. Hundley, A. E. Koshelev, L. N. Bulaevskii and A. J. Taylor, Phys.Rev. B 66, 012519 (2002).

10 R. D. Averitt and A. J. Taylor, J. Phys.: Condens. Matter 14, R1357-R1390 (2002).

11 J. Shan, F. Wang, E. Knoesel, M. Bonn, and T. F. Heinz, Phys. Rev. Lett. 90, 247401 (2003).

12 M. C. Beard, G. M. Turner, C. A. Schmuttenmaer, Phys. Rev. B 62, 15764 (2000).

13 K. P. H. Lui and F. A. Hegmann, Appl. Phys. Lett. 78, 3478 (2001).

14 R. D. Averitt, A. I. Lobad, C. Kwon, S. A. Trugman, V. K. Thorsmølle, and A. J. Taylor Phys. Rev. Lett. 87, 017401 (2001).

15 T.-I. Jeon and D. Grischkowsky, Phys. Rev. Lett. 78, 1106 (1997). 
16 F. A. Hegmann, R. R. Tykwinski, K. P. H Lui, J .E. Bullock and J. E. Anthony, Phys. Rev. Lett. 89, 227403 (2002).

17 C. Rønne, P.-O. Åstrand and S. R. Keiding, Phys. Rev. Lett. 82, 2888 (1999).

18 R. D. Averitt, G. Rodriguez, J. L. W. Siders, S. A. Trugman and A. J. Taylor, J. Opt. Soc. Am. B 17, 327 (2000).

19 V. Y. Butko, X. Chi, D. V. Lang and A. P. Ramirez, Preprint, cond-mat/0305402 (2003).
20 D. Moses, A. Dogariu and A. J. Heeger, Phys. Rev. B. 61, 9373 (2000).

${ }^{21}$ P. B. Miranda, D. Moses, and A. J. Heeger, Phys. Rev. B. 64, 08120(R) (2001).

22 W. Grauper, G. Cerullo, G. Lanzani, M. Nisoli, E. J. W. List, G. Leising and S. De. Selvistri, Phys. Rev. Lett. 81, 3259 (1998). 